\title{
DFNA5 attenuated in head and neck squamous cell carcinoma acquired with radio-resistance and associated with PD-L2
}

\section{Weiqiang Huang}

Southern Medical University Nanfang Hospital

\section{Lu Yuan}

Southern Medical University Nanfang Hospital

\section{Qiaocong Zheng}

Southern Medical University Nanfang Hospital

\section{Hua Pan}

Southern Medical University Nanfang Hospital

Mi Yang

Southern Medical University Nanfang Hospital

\section{Xixi Wu}

Southern Medical University Nanfang Hospital

\section{Longshan Zhang}

Southern Medical University Nanfang Hospital

\section{Benfu He}

421 Hospital of PLA

\section{Xiaoqing Wang}

Southern Medical University Nanfang Hospital

\section{Shaoqun Li}

Guangdong 999 Brain Hospital

\section{Yin Wang}

Southern Medical University Nanfang Hospital

\section{Feng Ye}

Southern Medical University Nanfang Hospital

Jian Guan ( $\nabla$ guanjian5461@163.com )

Nanfang hospital https://orcid.org/0000-0002-1913-1460

\section{Research}

Keywords: DFNA5, head and neck squamous cell carcinoma, PD-L2, radioresistance, PD-L1 
Posted Date: March 30th, 2020

DOl: https://doi.org/10.21203/rs.3.rs-19851/v1

License: (c) (i) This work is licensed under a Creative Commons Attribution 4.0 International License. Read Full License 


\section{Abstract}

Treatment of recurrent head and neck squamous cell carcinoma (HNSCC) after radiotherapy (RT) with immune checkpoint inhibitors (ICls) have attained certain success recently. However, some patients remained progressed and the total efficacy was finite, highlighting the need to identify new therapeutic options. DFNA5, also called GSDME, could switch chemotherapy drug induced-pyroptosis mediated by caspase-3. Presently, the relationship between DFNA5 and radiation in HNSCC remained unclear. Here, we showed that DFNA5 was up-regulated in HNSCC, and correlated with the expression of PD-L2, both of which reduced after the acquisition of radioresistance. Furthermore, PD-L1 was also tended to attenuate in radiation-resistant HNSCC. In sum, we showed that DFNA5 overexpressed in HNSCC and decreased with PD-L2 after attaining radiation-resistance, suggesting the potential role of DFNA5 as a possible combined target to amplify the immunotherapeutic efficacy in the future.

\section{Introduction}

Despite therapeutic advancements, the five-year survival rate of head and neck cancer (HNC) is less than $50 \%{ }^{[1]}$. As a majority of HNC, HNSCC poses a great threat to human being health. Crucially, RT exerting as an effective tool in local treatment has attained a great achievement for HNSCC, and one of most urgent challenges for it is the acquisition of irradiation-resistance. Afterwards, DFNA5 (Deafness, Autosomal Dominant 5), originally defined as a gene responsible for hearing loss, has been testified participating in chemotherapy drug-induced pyroptosis to increase chemosensitivity in recent years ${ }^{[2]}$. However, whether DFNA5 correlates with irradiation and radiosensitivity is elusive.

Recently, ICls has been approved for patients with recurrent and metastatic HNSCC by certain clinical trials, and partial patients have benefited from the immunotherapy greatly ${ }^{[3-5]}$. Meanwhile, we also noticed that a certain proportion of patients showed little response to pure ICls with disease progression. And the status of PD-L2 or other immune biomarkers associated with immune response remained unclear when HNSCC acquired the characteristic of radioresistance. Here, we showed that DFNA5 was upregulated in HNSCC and correlated with the expression level of PD-L2, both of which had a descent in recurrent HNSCC with radiation-resistance. Meanwhile, the level of PD-L1 also seemed to down-regulate in tissue not sensitive to irradiation. Thus, the phenomenon could partially explain why ICls targeted to PD-L1 failed to obviously improve prognosis for segmental irradiation-refractory HNSCC patients. Together, all data provided the possible viability of DFNA5 as a combined target with PD-L2 in HNSCC. And the relationship between DFNA5 and immune checkpoint related factors as well as radioresistance deserves further exploration in the future.

\section{Methods}

\section{Data availability and bioinformatics mining of DFNA5}


Gene expression RNA sequencing data of HNSCC cohort was downloaded from The Cancer Genome Atlas database (https://tcga-data.nci.nih.gov/tcga/tcgaDownload.jsp) in the year of 2015. Expression levels of DFNA5 were designated as high or low in relation to optimal expression via X-Tile software ${ }^{[6]}$. Data of GSEA6331 was attained from the NCBI Gene Expression omnibus (GEO, http://www.ncbi.nlm.nih.gov/geo/) and applied to Gene set enrichment analysis (GSEA) using GSEA software version 3.0. TISIDB analytical website (http://cis.hku.hk/TISIDB/index.php) was used for analyzing the interaction between DFNA5 and immunity as described ${ }^{[7]}$.

MiRDB and Targetscan databases were used to predict mircroRNAs that target DFNA $5^{[8]}$. Interacting proteins (IPs) of DFNA5 were predicted using the String database (https://string-db.org/). GSEA analysis of the gene sets responsible for these IPs were conducted via online analysis tool (https://amp.pharm.mssm.edu/Enrichr/). Transcription factors (TFs) of DFNA5 were predicted using PROMO database (http://alggen.Isi.upc.es/cgibin/promo_v3/promo/promoinit.cgi?dirDB=TF_8.3). Construction of related network was made via Cytoscape software 3.7.2 version. Evolutionary analysis of GSDM family was conducted via MEGA related software as described ${ }^{[9]}$.

\section{Cell culture and establishment of radioresistant cell line}

The human nasopharyngeal carcinoma cell line 5-8F was purchased from

Cell Bank of Type Culturen Collection of the Chinese Academy of Sciences (Shanghai Institute of Cell Biology) and cultured in 1640 (Gibco) supplemented with 10\% FBS (Gibco) and 1\% penicillin/streptomycin (Thermo Scientific). All cells were cultured in a humidified incubator containing $5 \% \mathrm{CO}_{2}$ at $37^{\circ} \mathrm{C}$.

The establishment of radioresistant cell line was performed as mentioned ${ }^{[10]}$. In brief, cells were treated with irradiation in the graded dose of 2, 4, 6, 8 and $10 \mathrm{~Gy}$ twice. The total IR dose of $60 \mathrm{~Gy}$ was given with the whole selection procedure being completed near 5 months. The final surviving $5-8 \mathrm{~F}$ cells were testified and defined as radioresistant $5-8 \mathrm{~F}$ cells and named $5-8 \mathrm{~F}-\mathrm{IRR}$. The parental $5-8 \mathrm{~F}$ cells were treated without irradiation. Cells within 5-10 passages were used for experiments once the cell line of 5-8F-IRR was established successfully.

\section{Clone formation assay}

Cells were seeded in six-well plates at various densities for $12 \mathrm{~h}$ and exposed to IR at doses of $0,2,4,6,8$ Gy. Cells were then cultured for an additional 12 to 14 days, and the surviving colonies containing over 50 cells were counted. Surviving fraction (SF) was estimated by the following formula: $\mathrm{SF}=$ (number of colonies formed / number of cells seeded $\times$ plating efficiency of the control group), where plating efficiency was calculated as the ratio between colonies observed and the number of cells plated. Doseresponse clonogenic survival curves were plotted on a log-linear scale.

\section{Western blotting analysis}


Cells were lysed with RIPA lysis buffer (Beyotime, Shanghai, China) supplemented with a protease and phosphatase inhibitor cocktail (Roche). 10\% SDS-PAGE was used and followed by blotting with the specific primary antibodies subsequently. Primary antibodies involved in this process included DFNA5 (13075-1-AP, Proteintech, USA), PD-L2 (18251-1-AP, Proteintech, USA), PD-L1 (66248-1-IG, Proteintech, USA), GAPDH (60004-1-IG, Proteintech, USA). GAPDH was used as internal reference.

\section{Virus transfection}

Lentivirus vectors containing DFNA5 and control were constructed and generated by Genechem Inc. (Shanghai, China). The lentiviral transfected cells were selected with puromycin (Clontech, USA). Stable infected cell lines were used for experiments.

\section{Immunohistochemistry (IHC) assay}

Human specimens derived from laryngopharynx and oral cavity were obtained from Nanfang Hospital, Southern Medical University. 11 cases of normal tissue and 20 cases of tumor specimens were acquired. Total staining processes were conducted according to manufacturer's instruction. Primary incubated antibodies included DFNA5 (13075-1-AP, Proteintech, USA), PD-L2 (18251-1-AP, Proteintech, USA), PD-L1 (66248-1-IG, Proteintech, USA). Results of IHC assay was evaluated on the basis of staining intensity and positively stained areas by two independent observers ${ }^{[9]}$. Positive cutoff value of $1 \%$ was used for the evaluation of PD-L2 and PD-L1 (positive $>1 \%$, negative $<1 \%$ ).

\section{Statistical analysis}

Statistical data analysis was performed using SPSS software 20.0 version. Paired/unpaired $t$-test was adopted for data between two experimental groups. Mann-Whitney tests were used to calculate the $P$ values for the quantification of IHC staining. Data were presented as the means \pm standard deviation (SD). Statistical significance was defined as the $P$-value was $<0.05$. ${ }^{\star} P<0.05 ; * \star P<0.01 ; * \star \star P<0.001$; $\star \star \star \star P<0.0001 ;$ ns, no significance.

\section{Results}

\section{Bioinformatic mining of DFNA5}

Despite the role of inducing pyroptosis has been reported, little is known regarding to the bioinformatic analysis of DFNA5. Here, we preliminarily performed related mining of DFNA5 ${ }^{[2]}$. Based on present researches, GSDM family consisted of six members, called GSDMA, GSDMB, GSDMC, GSDMD, GSDME (DFNA5) and DFNB59, respectively. Evolutionary analyses showed that GSDM family was divided into five clusters, of which DFNA5 and GSDMC were classified into same module (Fig 1A). TFs as well as the microRNAs that regulated DFNA5 were demonstrated (Fig 1B-D). Hsa-mir-582-5p was predicted to own conserved consequential pairing of DFNA5. Proteins that interacted with DFNA5 were acquired (Fig 1E), 
followed the GSEA of the gene sets that encoded these proteins. As shown, pathways associated with pyroptosis and DNA damage were enriched (Fig 1F).

\section{DFNA5 overexpressed in head and neck squamous carcinoma}

In order to explore the role of DFNA5 in HNSCC, we first analyzed the corresponding expression level in TCGA database. As shown (Fig 2A), DFNA5 mRNA expression was evidently increased in carcinoma compared with normal tissue. Similarly, the result was testified by GSE6631 dataset (Fig 2B). And patients with high DFNA5 expression correlated with worse overall survival (Fig 2C). Importantly, the expression of DFNA5 was associated with the status of Human papilloma virus (HPV) negatively (Fig 2D). And we also detected the expression of DFNA5 with clinical samples via IHC that DFAN5 was highly expressed in HNSCC carcinoma tissue (Fig 2E-F).

To further uncover the biological characteristics of DFNA5 in HNSCC, we made a GSEA based on the expression level of DFNA5 with GSE6631 dataset, of which the principle component analysis was carried out (Fig S1A). The results of GSEA showed (Fig S1B-C) that biological process of DNA repair as well as TGF- $\beta$ signaling were attained.

\section{DFNA5 correlated with radiation}

Irradiation as one effective therapeutic tool plays a crucial role in HNC local treatment ${ }^{[11-12]}$. Additionally, previous evidence had revealed the remarkable alteration of DFNA5 induced by gamma-ray radiation at the mRNA level[ ${ }^{[13]}$. Therefore, we speculated that DFNA5 was associated with response to radiation possibly. Expectedly, further biological analysis showed that DFNA5 correlated with pathways regarding to DNA repair and response to ionizing radiation (Fig S1B-C, Fig 3A). Next, we constructed radioresistant cell line and found that the level of DFNA5 protein decreased significantly after the subsequent acquisition of characteristic to resist radiation when compared with control group (Fig 3B-D). Consistently, we validated the viewpoint by IHC that DFNA5 had a tendency to decrease in irradiationresistant HNSCC tissue (Fig 3E, Table 1). 
Table 1. Clinical Characteristics of Patients treated with irradiation

\begin{tabular}{|c|c|}
\hline Characteristic & All patients (12) \\
\hline \multicolumn{2}{|l|}{ Age (years) } \\
\hline Mean & 54 \\
\hline Range & $40-71$ \\
\hline \multicolumn{2}{|l|}{ Sex } \\
\hline Male & 10 \\
\hline Female & 2 \\
\hline \multicolumn{2}{|l|}{ Smoking history } \\
\hline Yes & 6 \\
\hline No & 6 \\
\hline \multicolumn{2}{|c|}{ Location of primary tumour } \\
\hline Nasopharynx & 1 \\
\hline Oropharynx & 2 \\
\hline Oral cavity & 2 \\
\hline Tongue & 3 \\
\hline Larynx & 2 \\
\hline Hypopharynx & 2 \\
\hline \multicolumn{2}{|l|}{ Recurrence } \\
\hline Yes & 7 \\
\hline No & 5 \\
\hline
\end{tabular}

\section{DFNA5 correlated with the level of PD-L2}

Currently, immunotherapy has appeared as a new frontier of cancer treatment. Yet, the overall response rate for recurrent or metastatic HNSCC was restricted ${ }^{[3-4]}$. Given the fact that DFNA5 interacted with irradiation and related to HNSCC clinically, we supposed whether DFNA5 might function as a target combined with immunotherapy to further amplify clinical benefit. Therefore, we initially explored the relationship between DFNA5 and immune checkpoint related factors. As demonstrated, PD-1 ligation pathway was enriched (Figure 4A). Further analysis revealed that DFNA5 correlated with programmed death ligand-2 (PD-L2) that the expression of PD-L2 increased following the overexpression of DFNA5 (Fig 4B-E). Significantly, PD-L2 was highly expressed in HNSCC carcinoma which was consistent with the alteration of DFNA5 based on TCGA database (Fig S2A). Moreover, members of TGF- $\beta /$ TGF- $\beta$ R family was demonstrated to correlate with the level of DFNA5 (Fig S2B-C). Besides, reduced expression of DFNA5 in vitro or in radioresistant HNSCC was observed when compared with radiosensitive HNSCC carcinoma (Fig 4D, 4F). And among radiation-resistant HNSCC, three cases were PD-L2 positive while the other four cases were PD-L2 negative. Additionally, the descent of PD-L1 expression was also observed in vitro and in recurrent patients ever treated with radiation that four patients were negative while the others were positive (Fig S2D-E). Characteristics of radiation-resistant carcinoma was displayed in Table 1. 


\section{Discussion}

Despite rapid advances have achieved as regards the treatment for HNC, the five-year survival rate of advanced HNSCC is less than $50 \%$, and the metastatic event or recurrent tumors growing from radiationresistant carcinoma cells of HNSCC remains unsolved ${ }^{[1][14]}$. According to latest statistic published in the year of $2018, \mathrm{HNC}$ resulted in more than 336,000 cancer-related deaths worldwide per year ${ }^{[15]}$. Thus, it's urgent to disclose feasible targets to improve patients' prognosis.

DFNA5 was originally known as a gene responsible for hearing loss ${ }^{[13]}$. Recently, its role of mediating pyroptosis induced by chemotherapy was uncovered. Besides, it's reported that DFNA5 was unusual in several certain solid cancer ${ }^{[16]}$. However, the emerging roles and functions of DFNA5 in HNSCC were unclear. According to our research, DFNA5 was overexpressed in HNSCC. It's well known that HPV status had a significant impact on outcome and response to treatment for HNC patients. Further analysis revealed that the expression of DFNA5 was correlated with the status of HPV negatively, which was consistent with the fact that patients with high expression of DNFA5 and HPV-negative displayed worse prognosis $^{[14]}$.

Crucially, RT as an effective tool plays a pivotal role in local treatment for HNC. Shown the fact that DFNA5 overexpressed in HNSCC, the specific relationship between DFNA5 and RT remained elusive. Based on present research, DFNA5 was reported to participated in the process of DNA damage ${ }^{[13]}$. This was consistent with our biological analysis that DFNA5 closely related to DNA repair reaction as well as irradiation response. Furthermore, the predicted IPs of DFNA5 also related to DNA damage. Among these IPs, p53 was one of most known proteins that emerged in the process of DNA repair and the regulation of cell apoptosis. In vitro, DFNA5 was decreased in radioresistant cell line, which was confirmed by IHC with radiation-resistant HNSCC tissue.

Clinically, the incidence of recurrence and distant metastasis post-treatment resulted in the main HNSCCrelated death. Convenient therapies failed to benefit these kind of patients obviously. Fortunately, the appearance of ICls amplified the clinical benefit of these HNSCC patients and improved their prognosis significantly, when compared with convenient treatments including platinum-based drugs, drugs targeting to $\mathrm{EGFR}^{[17-18]}$. However, the total efficacy of single immunotherapy remained to be improved. Simultaneously, the subgroup analysis of irradiation-resistant HNSCC was undefined. Therefore, it's meaningful to take combination therapy for these kind of patients. The primary task was to search for potential targets enabling to interact with immune checkpoint related factors. Surprisingly, positive correlation between DFNA5 and PD-L2 was revealed according to our research, both of which were high expression in HNSCC carcinoma. Meanwhile, TGF- $\beta$ /TGF- $\beta$ R family also positively related to DFNA5 expression. Moreover, PD-L2 as well as PD-L1 tended to decline in locally recurrent HNSCC ever received accuracy radiotherapy, which was partially in line with the outcome that recurrent HNSCC patients applied to immunotherapy had limited efficacy. 
In sum, DFNA5 highly expressed in HNSCC, and the expression level got lessened upon the acquisition of radiation-resistance, suggesting the possibility for DFNA5 as a radiosensitive marker. Given the fact that

DFNA5 enabled to mediate pyroptosis which involved the release of inflammatory factors ${ }^{[19]}$, we speculated that DFNA5 had the potential to arouse immune-related reaction in tumor microenvironment. Further analyses showed that DFNA5 actually correlated with immune related objects, such as PD-L2 and TGF- $\beta$ family. Unlike PD-L1, PD-L2 has emerged as an area of exploration, and it was reported to be a potential therapeutic target ${ }^{[20]}$. Beyond that, recent emerging evidence has shown that TGF- $\beta$ would attenuate anti-tumor immune response produced by blockade of PD-L $1^{[21]}$. Additionally, it's well known that RT has a great impact on systematic immunity ${ }^{[12]}$ and our study integrated RT and DFNA5 as well as PD-L2 primarily. All mentioned above provided the feasibility for DFNA5 to function as a potential target cooperated with current immunotherapy to amplify relative efficacy. And more clinical samples are needed to further illustrate the relationship between DFNA5 and PD-L2 in HNSCC. Based on emerging evidence, our next plan is to investigate the mechanisms responsible for the alteration of DFNA5 and regulation of PD-L2 after irradiation, understanding how predicted has-mir-582-5p regulates the activity of DFNA5, illustrating the possibility of DFNA5 used as a coordinative target to enhance immunotherapy curative effect for cancer treatment in the future.

\section{Conclusion}

DFNA5 was up-regulated in HNC and down-regulated in radioresistant tissue. Biological analysis showed that DFNA5 associated with PD-L2, a ligand for PD-1, suggesting the potential role in adjusting immunity. Immunotherapy was demonstrated as a promising anti-tumor tool in recurrent HNC treated with irradiation in recent years. However, the effect remained to be improved. Recurrent HNC ever treated with irradiation usually acquired the characteristic of radioresistance. Correspondingly, our research revealed that DFNA5 as well as PD-L2 were lower expression in radio-resistance HNC tissues, highlighting the potential function of DFNA5 as a target in amplifying the effect of immunotherapy.

\section{Declarations}

\section{Ethics approval and consent to participate}

The study was approved by our institution.

\section{Consent for publication}

The consent for publication was obtained from every patient.

\section{Availability of data and materials}

The datasets involved in the current study are available from the corresponding author on reasonable request. 


\section{Competing interests}

The authors declared no competing interests.

\section{Funding}

The study was supported by National Natural Science Foundation of China (NO. 81602685, 81672992); Clinical Research Startup Program of Southern Medical University by High-level University Construction Funding of Guangdong Provincial Department of Education (LC2019ZD008); Clinical Research Startup Program of Southern Medical University by High-level University Construction Funding of Guangdong Provincial Department of Education (2018CR021); Health \& Medical Collaborative Innovation Project of Guangzhou City, China (201803040003); the Natural Science Foundation of Guangdong Province (NO. 2017A030313486); Guangzhou Science and Technology Plan Project (201707010025) and CAMS Innovation Fund for Medical Sciences (CIFMS, 2016-I2M-1-017 and 2017-I2M-B\&R-13).

\section{Authors' contributions}

Weiqiang Huang, Lu Yuan, Qiaocong Zheng analysed and interpreted all data. Hua Pan, Mi Yang, Xixi Wu, Longshan Zhang and Benfu He collected all data; Xiaoqing Wang; Shaoqun Li; Yin Wang were major contributors in writing the manuscript. All authors read and approved the final manuscript

\section{Acknowledgments}

We thank Department of Otolaryngology, Department of Stomatology, Nanfang Hospital for providing clinical samples.

\section{Abbreviations}

DFNA5: deafness, autosomal dominant 5

GSDM: gasdermin

ICls: immune checkpoint inhibitors

HNSCC: head and neck squamous cell cancer

HNC: head and neck cancer

$\mathrm{RT}$ : radiotherapy

PD-L1: programmed cell death 1 ligand 1

PD-L2: programmed cell death 1 ligand 2

TGF: transforming growth factor 
TCGA: The Cancer Genome Atlas

GSEA: gene set enrichment analysis

GEO: Gene Expression omnibus

TFs: transcription factors

IPs: interacting proteins

IHC: immunohistochemistry

\section{References}

[1] Haddad R. I., Shin D. M. Recent advances in head and neck cancer. N Engl J Med. 2008. 359(11). 11431154

[2] Wang Y., Gao W., Shi X., Ding J., Liu W., He H., Wang K., Shao F. Chemotherapy drugs induce pyroptosis through caspase-3 cleavage of a gasdermin. Nature. 2017. 547(7661). 99-103

[3] Fang W., Yang Y., Ma Y., Hong S., Lin L., He X., Xiong J., Li P., Zhao H., Huang Y., Zhang Y., Chen L., Zhou N., Zhao Y., Hou X., Yang Q., Zhang L. Camrelizumab (SHR-1210) alone or in combination with gemcitabine plus cisplatin for nasopharyngeal carcinoma: results from two single-arm, phase 1 trials. Lancet Oncol. 2018. 19(10). 1338-1350

[4] Chow LQM, Haddad R., Gupta S., Mahipal A., Mehra R., Tahara M., Berger R., Eder J. P., Burtness B., Lee S. H., Keam B., Kang H., Muro K., Weiss J., Geva R., Lin C. C., Chung H. C., Meister A., Dolled-Filhart M., Pathiraja K., Cheng J. D., Seiwert T. Y. Antitumor Activity of Pembrolizumab in Biomarker-Unselected Patients With Recurrent and/or Metastatic Head and Neck Squamous Cell Carcinoma: Results From the Phase Ib KEYNOTE-012 Expansion Cohort. J Clin Oncol. 2016. 34(32). 3838-3845

[5] Ferris R. L., Blumenschein G. Jr, Fayette J., Guigay J., Colevas A. D., Licitra L., Harrington K., Kasper S., Vokes E. E., Even C., Worden F., Saba N. F., Iglesias Docampo LC, Haddad R., Rordorf T., Kiyota N., Tahara M., Monga M., Lynch M., Geese W. J., Kopit J., Shaw J. W., Gillison M. L. Nivolumab for Recurrent Squamous-Cell Carcinoma of the Head and Neck. N Engl J Med. 2016. 375(19). 1856-1867

[6] Pan C., Wang X., Chen W., Tao C., Xu X., Jin L., Chen Y., Zhu L., Zhou L., Pan Z. Reevaluation of glypican-3 as a prognostic marker in HCC using X-tile software. Med Oncol. 2015. 32(1). 359

[7] Ru B., Wong C. N., Tong Y., Zhong J. Y., Zhong SSW, Wu W. C., Chu K. C., Wong C. Y., Lau C. Y., Chen I., Chan N. W., Zhang J. TISIDB: an integrated repository portal for tumor-immune system interactions. Bioinformatics. 2019. 35(20). 4200-4202 
[8] Fan Y., Zhang L., Sun Y., Yang M., Wang X., Wu X., Huang W., Chen L., Pan S., Guan J. Expression profile and bioinformatics analysis of COMMD10 in BALB/C mice and human. Cancer Gene Ther. 2019.

[9] Yang M., Huang W., Sun Y., Liang H., Chen M., Wu X., Wang X., Zhang L., Cheng X., Fan Y., Pan H., Chen L., Guan J. Prognosis and modulation mechanisms of COMMD6 in human tumours based on expression profiling and comprehensive bioinformatics analysis. Br J Cancer. 2019. 121(8). 699-709

[10] Li G., Liu Y., Su Z., Ren S., Zhu G., Tian Y., Qiu Y. MicroRNA-324-3p regulates nasopharyngeal carcinoma radioresistance by directly targeting WNT2B. Eur J Cancer. 2013. 49(11). 2596-2607

[11] Tian Y. M., Tian Y. H., Zeng L., Liu S., Guan Y., Lu T. X., Han F. Prognostic model for survival of local recurrent nasopharyngeal carcinoma with intensity-modulated radiotherapy. Br J Cancer. 2014. 110(2). 297-303

[12] Huang Weiqiang, Fan Yao, Cheng Xiaoya, Liang Huazhen, Pan Hua, Xiao Ting, Chen Min, Guan Jian. A preliminary Study on the Effect of Head and Neck Chemoradiotherapy on Systematic Immunity. DOSERESPONSE. 2019. 17(15593258198841864)

[13] Masuda Y., Futamura M., Kamino H., Nakamura Y., Kitamura N., Ohnishi S., Miyamoto Y., Ichikawa H., Ohta T., Ohki M., Kiyono T., Egami H., Baba H., Arakawa H. The potential role of DFNA5, a hearing impairment gene, in p53-mediated cellular response to DNA damage. J Hum Genet. 2006. 51(8). 652-664

[14] Skvortsov S., Dudas J., Eichberger P., Witsch-Baumgartner M., Loeffler-Ragg J., Pritz C., Schartinger V. H., Maier H., Hall J., Debbage P., Riechelmann H., Lukas P., Skvortsova I. Rac1 as a potential therapeutic target for chemo-radioresistant head and neck squamous cell carcinomas (HNSCC). Br J Cancer. 2014. 110(11). 2677-2687

[15] Bray F., Ferlay J., Soerjomataram I., Siegel R. L., Torre L. A., Jemal A. Global cancer statistics 2018: GLOBOCAN estimates of incidence and mortality worldwide for 36 cancers in 185 countries. CA Cancer $J$ Clin. 2018. 68(6). 394-424

[16] Op De Beeck K., Van Camp G., Thys S., Cools N., Callebaut I., Vrijens K., Van Nassauw L., Van Tendeloo V. F., Timmermans J. P., Van Laer L. The DFNA5 gene, responsible for hearing loss and involved in cancer, encodes a novel apoptosis-inducing protein. Eur J Hum Genet. 2011. 19(9). 965-973

[17] Harrington K. J., Ferris R. L., Blumenschein G. Jr, Colevas A. D., Fayette J., Licitra L., Kasper S., Even C., Vokes E. E., Worden F., Saba N. F., Kiyota N., Haddad R., Tahara M., Grunwald V., Shaw J. W., Monga M., Lynch M., Taylor F., DeRosa M., Morrissey L., Cocks K., Gillison M. L., Guigay J. Nivolumab versus standard, single-agent therapy of investigator's choice in recurrent or metastatic squamous cell carcinoma of the head and neck (CheckMate 141): health-related quality-of-life results from a randomised, phase 3 trial. Lancet Oncol. 2017. 18(8). 1104-1115 
[18] Cohen EEW, Soulieres D., Le Tourneau C., Dinis J., Licitra L., Ahn M. J., Soria A., Machiels J. P., Mach N., Mehra R., Burtness B., Zhang P., Cheng J., Swaby R. F., Harrington K. J. Pembrolizumab versus methotrexate, docetaxel, or cetuximab for recurrent or metastatic head-and-neck squamous cell carcinoma (KEYNOTE-040): a randomised, open-label, phase 3 study. Lancet. 2019. 393(10167). 156-167

[19] Rogers C., Fernandes-Alnemri T., Mayes L., Alnemri D., Cingolani G., Alnemri E. S. Cleavage of DFNA5 by caspase-3 during apoptosis mediates progression to secondary necrotic/pyroptotic cell death. Nat Commun. 2017. 8. 14128

[20] Zhao S. G., Lehrer J., Chang S. L., Das R., Erho N., Liu Y., Sjostrom M., Den RB, Freedland S. J., Klein E. A., Karnes R. J., Schaeffer E. M., Xu M., Speers C., Nguyen P. L., Ross A. E., Chan J. M., Cooperberg M. R., Carroll P. R., Davicioni E., Fong L., Spratt D. E., Feng F. Y. The Immune Landscape of Prostate Cancer and Nomination of PD-L2 as a Potential Therapeutic Target. J Natl Cancer Inst. 2019. 111(3). 301-310

[21] Mariathasan S., Turley S. J., Nickles D., Castiglioni A., Yuen K., Wang Y., Kadel EE III, Koeppen H., Astarita J. L., Cubas R., Jhunjhunwala S., Banchereau R., Yang Y., Guan Y., Chalouni C., Ziai J., Senbabaoglu Y., Santoro S., Sheinson D., Hung J., Giltnane J. M., Pierce A. A., Mesh K., Lianoglou S., Riegler J., Carano RAD, Eriksson P., Hoglund M., Somarriba L., Halligan D. L., van der Heijden M. S., Loriot Y., Rosenberg J. E., Fong L., Mellman I., Chen D. S., Green M., Derleth C., Fine G. D., Hegde P. S., Bourgon R., Powles T. TGFbeta attenuates tumour response to PD-L1 blockade by contributing to exclusion of T cells. Nature. 2018. 554(7693). 544-548

\section{Figures}


A
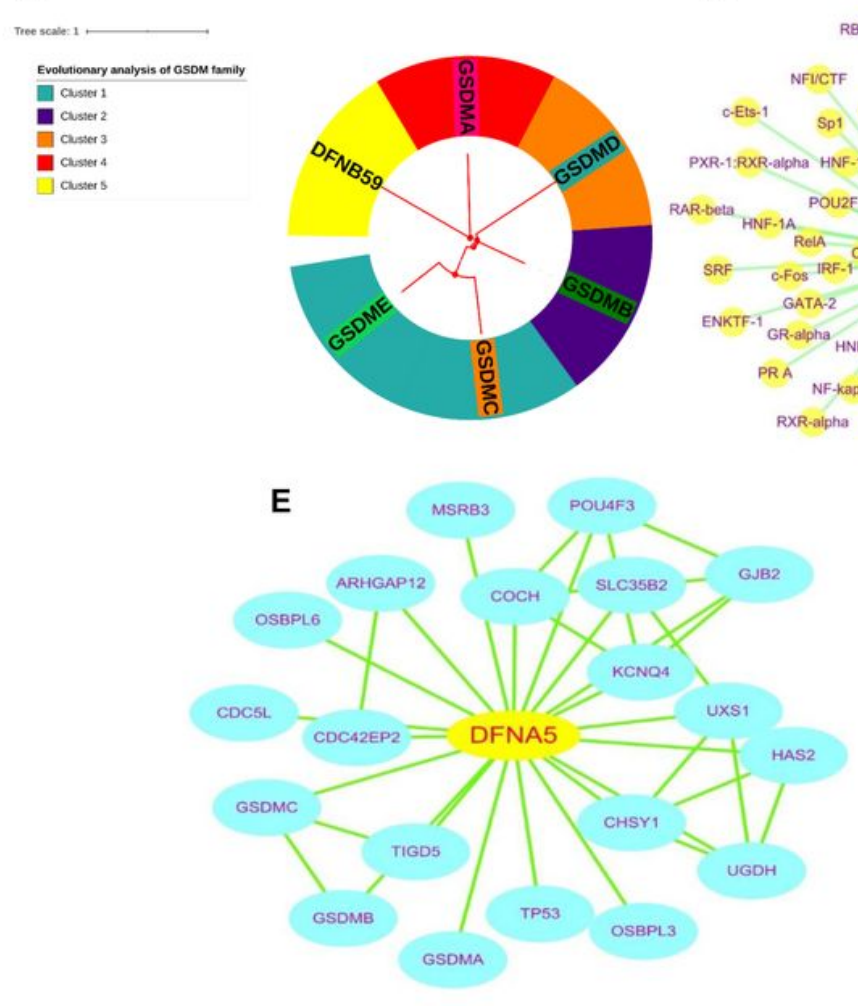

B

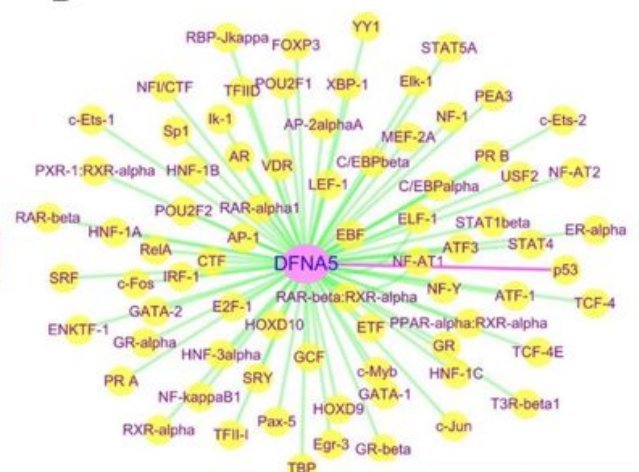

C

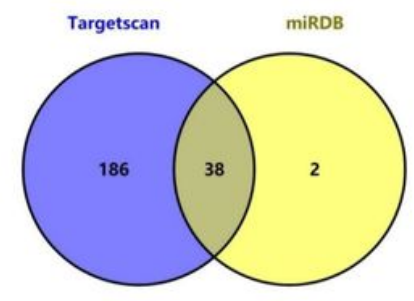

D

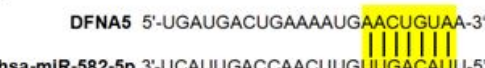

F
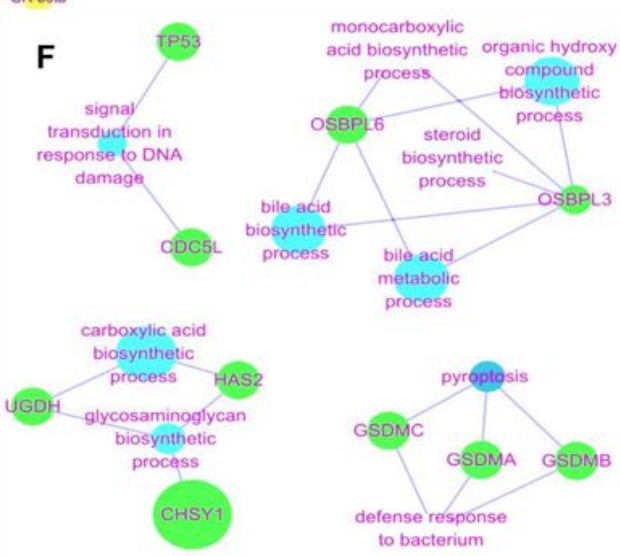

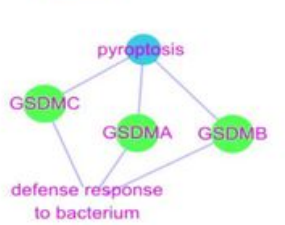

\section{Figure 1}

Bioinformatic mining of DFNA5 gene. (A) Evolutionary analyses of GSDMD family. (B) The network of TFs of DFNA5. (C-D) Predicted microRNA and the predicted pairing sequencing of DFNA5. (E-F)The network of IPs interacted with DFNA5 and the GSEA of genes set encodeing these proteins. 

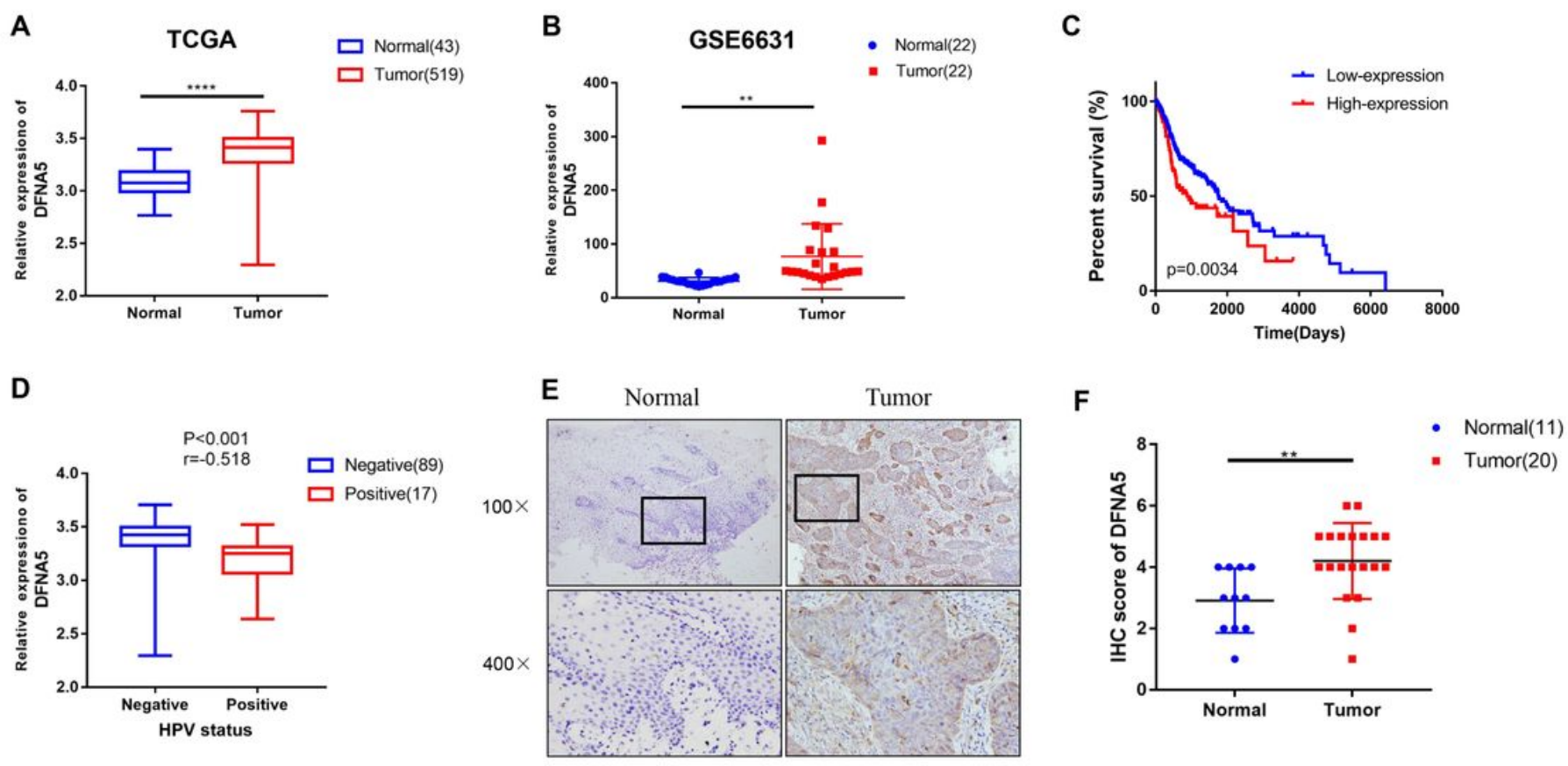

\section{Figure 2}

DFNA5 dysregulated in HNSCC. (A-B) DFNA5 overexpressed in HNSCC. (C) High expression of DFNA5 predicted worse prognosis. (D) DFNA5 correlated with HPV status negatively. (E-F) IHC staining of DFNA5 in HNSCC. The representing images were shown. *, $p<0.05 ; * \star, p<0.01 ; * \star *, p<0.001 ; * \star \star \star, p<0.0001$. 
A
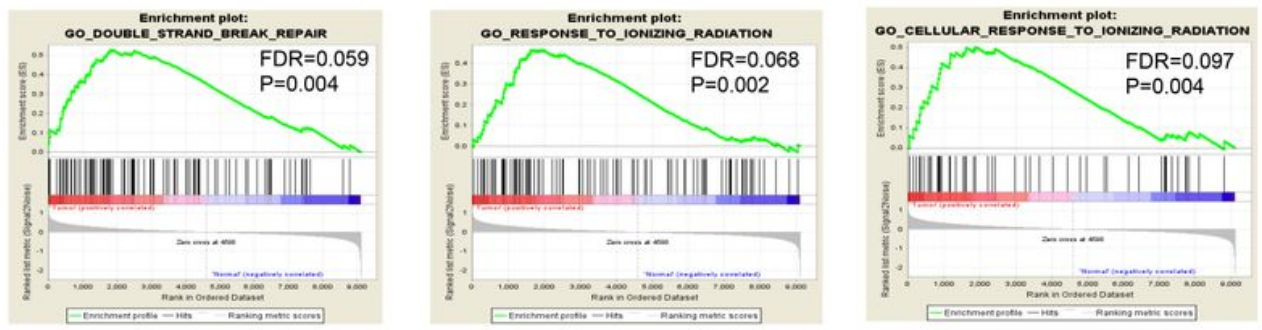

B

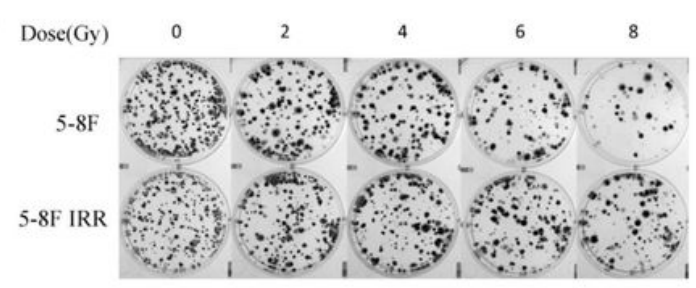

C
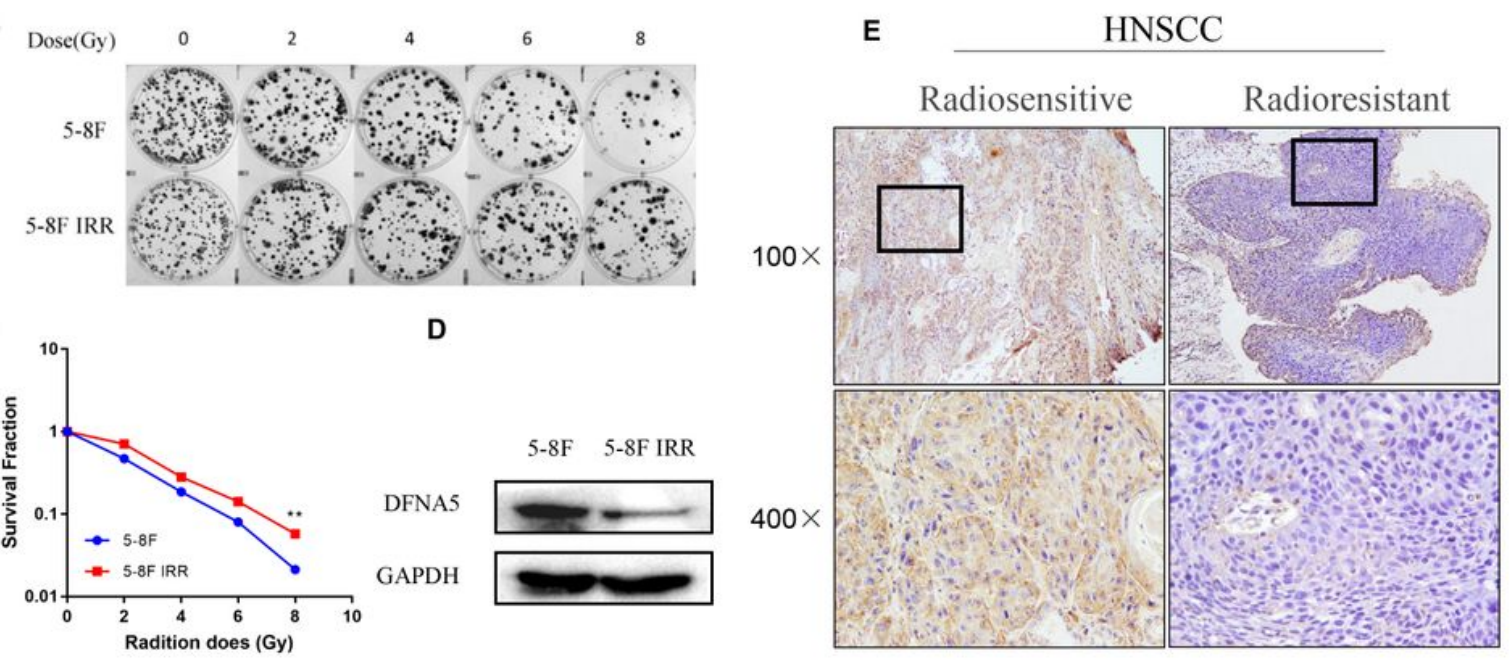

\section{Figure 3}

DFNA5 down-regulated in radioresistant HNSCC. (A) DNA repair and response to irradiation were enriched. (B-C) The establishment of radiation-resistant cell line and the survival curve of it were shown. (D-E) DFNA5 expression was reduced in radiation-resistant cell line and radioresistant HNSCC tissues. The representing images were shown.

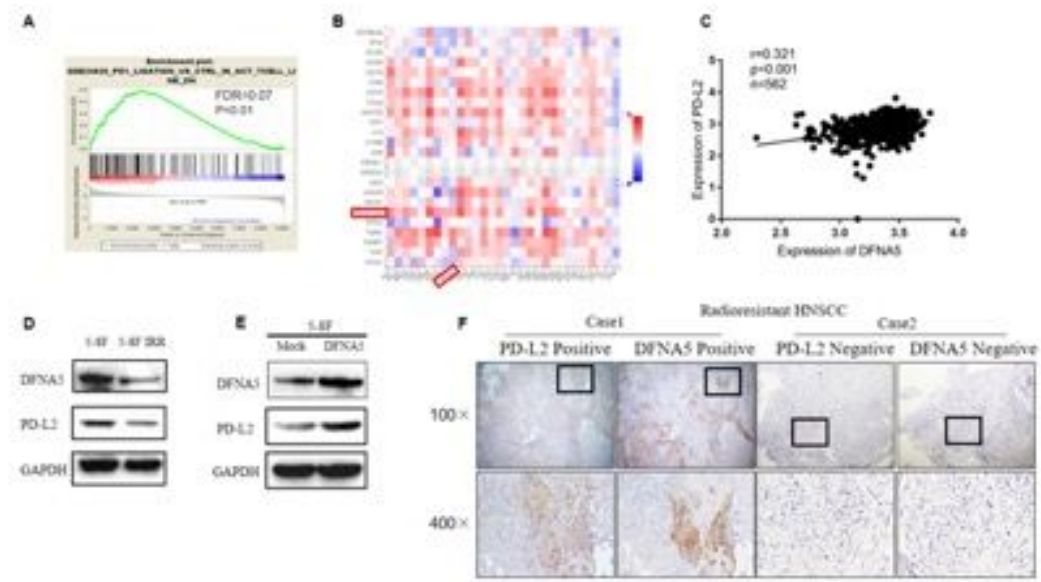

\section{Figure 4}

DFNA5 correlated with PD-L2. (A) PD-1 ligation was enriched. (B-C) DFNA5 correlated with PD-L2. (D) Both expression of DFNA5 and PD-L2 reduced in radiation-resistant 5-8F cell line. (E) And the 
overexpression of DFNA5 increase the expression level of PD-L2. (F) IHC of DFNA5 and PD-L2 in radioresistant HNSCC samples. The representing images were shown.

\section{Supplementary Files}

This is a list of supplementary files associated with this preprint. Click to download.

- S1.pdf

- S2.pdf 